\title{
Ist die Ordnungsökonomik zukunftsfähig?
}

\author{
LARS P. FELD UND EKKEHARD A. KÖHLER ${ }^{*}$
}

\section{Constitutional economics: $A$ viable approach?}

This paper deals with the future of "Ordnungsökonomik" (constitutional economics) within economic science regarding its dual character as a positive science on the one side and as a normative analysis trying to give policy advice on the other. From the perspective of normative individualism the criteria of legitimacy of individual consent or the ideals of governance of citizen and consumer sovereignty can serve as a basis for a modern adjustment of constitutional economics. In accordance with the constitutional economics approach of James Buchanan citizens' interest will therefore be used as reference criteria. After an overview on the recent methodological debate ("Methodensrteit") among economists we will discuss, based on the constitutional economics approach, recent arguments on the future development of constitutional economics. Finally we will present our own position concerning the future of the constitutional economics approach.

Keywords: constitutional economics, methodological debate, theoretical development, critics, further development, Werturteilsstreit, Ordnungsökonomik

\section{Einleitung}

Die Finanz- und Wirtschaftskrise der Jahre 2007 und 2008 löste eine intensive Debatte über die „Zukunft des Kapitalismus“ aus, die insbesondere in den angelsächsischen Ländern und in Deutschland in einen Diskurs über eine Neuorientierung der Wirtschaftswissenschaften mündete (Kirchgässner 2009). Während es in Großbritannien keine andere als die Queen des Vereinigten Königreichs war, die an der London School of Economics zur Selbstüberprüfung der Profession aufrief (Pierce 2008), entbrannte in den Vereinigten Staaten ein bis heute andauernder Streit darüber, wie der Staat bestmöglich auf die Krise zu reagieren habe (Krugman 2009: 36, 2011: A21). In Deutschland war es wieder einmal die Methode, die zum Hauptgegenstand der Reflexion wurde. So entwickelte sich aus einer Debatte um die Zukunft der deutschen Wirtschaftswissenschaften eine Kontroverse, die als ,jüngster“ oder „neuer“ Methodenstreit in die Literatur eingegangen ist (Caspari und Schefold 2011). Freilich ist dieser Methodenstreit kein rein deutsches Phänomen und zieht auch in der angelsächsischen Welt seine Kreise (Buchanan 2010).

Prof. Dr. Lars P. Feld, Walter Eucken Institut, Goethestraße 10, 79100 Freiburg i. Br., Tel.: +49 (0)761-79097-0, E-Mail: feld@walter-eucken-institut.de, Forschungsschwerpunkte: Wirtschaftspolitik, Finanzwissenschaft, Neue Politische Ökonomie.

Dipl.-Vw. Ekkehard A. Köhler, Walter Eucken Institut, Goethestraße 10, 79100 Freiburg i. Br., Tel.: +49 (0)761-79097-11, E-Mail: koehler@walter-eucken-institut.de, Forschungsschwerpunkte: Institutionenökonomik, Geldtheorie. 
Die gesellschaftliche Auseinandersetzung führte hingegen zu einer deutlich geringeren Akzeptanz der Marktwirtschaft in einer Vielzahl von Ländern. Eine jüngere Umfrage, die in 25 Industrie- und Schwellenländern durchgeführt wurde, belegt diesen Rückgang der Zustimmung zum marktwirtschaftlichen System in den USA, Großbritannien und Frankreich. ${ }^{1}$ Während 2002 noch etwa 80 Prozent der befragten Amerikaner die Marktwirtschaft befürworteten, sank dieser Anteil im Jahr 2010 auf 59 Prozent. In Großbritannien ging die Akzeptanz der Marktwirtschaft im gleichen Zeitraum leicht von knapp über auf knapp unter 60 Prozent zurück. In Frankreich, das 2002 schon auf dem relativ niedrigen Akzeptanzniveau von 42 Prozent der Befragten lag, reduzierte sich das Ansehen der Marktwirtschaft hingegen auf 30 Prozent. Lediglich Spanien geht diesem Trend entgegen. Dort stieg die Akzeptanz des marktwirtschaftlichen Systems von 37 Prozent im Jahr 2002 auf 51 Prozent im Jahr 2010. Am stärksten akzeptiert ist das marktwirtschaftliche System heute in China und Brasilien mit 68 Prozent der Befragten und in Deutschland, das mit 69 Prozent das Länderranking anführt. In Deutschland nahm die Zustimmung zur Marktwirtschaft von 2002 bis 2007 gleichwohl ab und ist erst seitdem wieder angestiegen. Kapitalismuskritik findet sich in weiten Teilen der deutschen Bevölkerung, insbesondere unter Intellektuellen (Wissenschaftlicher Beirat beim Bundesministerium für Wirtschaft und Technologie 2009; Schirrmacher 2010).

Damit unterscheidet sich das Resümee der gesellschaftlichen Reflexion vom Konsens, der trotz des Methodenstreits noch immer in den Wirtschaftswissenschaften vorherrscht: Insbesondere theoretische und empirische Erkenntnisse über die Funktionseigenschaften von (marktwirtschaftlichen) Institutionen lassen die Marktwirtschaft (und mit ihr die Demokratie) als günstigste Formen der Koordination individueller Entscheidungen erscheinen (Voigt 1997, 2011; Kirchgässner 2009; Vanberg 2011). Weder die Finanz- und Wirtschaftskrise noch der Methodenstreit haben diesen Konsens in den Wirtschaftswissenschaften bislang in Frage gestellt. Der Vergleich zwischen den gesellschaftlichen und wirtschaftswissenschaftlichen Debatten legt folglich Unterschiede in der Entwicklungsrichtung beider Diskurse offen, die hinsichtlich ihrer Konsequenz für die Wirtschaftswissenschaften nicht ernst genug genommen werden können: Angesichts dieses Auseinanderlaufens beider Reflexionsprozesse könnte es künftig für Wirtschaftswissenschaftler erheblich schwieriger sein, gesellschaftliche Zustimmung für ihre Handlungsempfehlungen zu erhalten.

Diese Beobachtung sollte der Wirtschaftswissenschaft Anlass sein, sich ihrer gesellschaftlichen Verantwortung zu erinnern, wie dies im Methodenstreit bereits gefordert wurde (Goldschmidt et al. 2009; Haucap 2009). Herbert Giersch $(1993,1994)$ sprach in solchen Zusammenhängen von einer Bringschuld der Ökonomen, den Bürger über wirtschaftspolitische Zusammenhänge zu informieren: „Außer dem Warnen vor Fehlentwicklungen in der Zukunft gehört zu den Aufgaben das Aufdecken von Fehlern, die sich korrigieren lassen, und von Verbesserungsmöglichkeiten allgemein“ (Giersch

Im Jahr 2010 befragte Globescan 12.884 Personen. Siehe Globescan (2011), Sharp Drop in American Enthusiasm for Free Market, Poll Shows, http://www.globescan.com/news_ archives/radar10w2_free_market/ (Download am 9. August 2011). In seiner Ausgabe vom 9. April wurden die Ergebnisse vom Economist aufgegriffen. Siehe „Market of Ideas“, The Economist 399 (8728), April 9th, 2011, S. 60. 
1994: 246). Letztlich gehöre dazu auch die Möglichkeit eines Wissenschaftsversagens: „Wo sind wir blind infolge traditioneller Vorurteile und Tabus, wo sind wir geblendet von modischen Modellvorstellungen?" (Giersch 1994: 246). Tröstlich sei dabei, dass die Wirtschaftswissenschaft ein offenes System sei. Damit lehnt sich Giersch an Karl Popper an, der jedoch auch gezeigt hat, „dass [...] holistische Ansprüche nicht einlösbar sind und häufig nur ideologische Standpunkte kaschieren“ (Kirchgässner 2009a). Die Wirtschaftswissenschaften können daher, wenn sie als Wissenschaft ernst genommen werden wollen, nicht die einzig gültige Wahrheit verkünden, sondern müssen zwischen (empirisch fundierten) Klugheitsargumenten abwägen und diesen Erkenntnisprozess auf zugängliche Weise dokumentieren. Die von Giersch eingeforderte Bringschuld muss daher mit einem Methodenpluralismus einhergehen, um einen möglichst breiten Erkenntnisgewinn für den Bürger zu erzielen.

In diesem Beitrag setzen wir uns mit der zukünftigen Positionierung der Ordnungsökonomik innerhalb der Wirtschaftswissenschaften in dem zuvor angelegten Spannungsfeld zwischen positiver Analyse und normativer, auf wirtschaftspolitische Beratung zielender Disziplin auseinander. Ausgangspunkt der Betrachtung ist ein streng normativ-individualistischer, gemäß dem der Bürger als Individuum Legitimationsquelle für politische Handlungsempfehlungen ist. Ganz im Sinne des verfassungsökonomischen Forschungsansatzes von James Buchanan werden die Bürgerinteressen als Referenzkriterium herangezogen. Um eine dem normativen Individualismus entsprechende Referenzgröße zu finden, bieten sich das Legitimationskriterium der individuellen Zustimmung oder die Steuerungsideale der Bürger- und Konsumentensouveränität an. Im Weiteren gehen wir wie folgt vor: Zunächst wird ein Überblick über den aktuellen Stand der wirtschaftswissenschaftlichen Reflexion - dem Methodenstreit - geliefert und eine kurze Einordnung vorgenommen (Abschnitt 2). Anschließend diskutieren wir jüngste Beiträge zur zukünftigen Ausrichtung der Ordnungsökonomik vor dem Hintergrund des verfassungsökonomischen Forschungsprogramms (Abschnitt 3). In einem dritten Schritt wird unsere Perspektive zur Weiterentwicklung des ordnungsökonomischen Forschungsansatzes dargestellt (Abschnitt $4)$.

\section{Die jüngere Kritik an der Ordnungsökonomik und der neuere Methodenstreit}

Eine methodische Auseinandersetzung in der Ordnungsökonomik war längst überfällig. Während des Systemwettbewerbs zu Zeiten des Kalten Krieges war es in den Wirtschaftswissenschaften allgemein akzeptiert, wirtschaftspolitische Handlungsempfehlungen mittels externer Werturteile zu legitimieren. ${ }^{2}$ Privateigentum, Vertragsfreiheit und die Notwendigkeit eines staatlichen Ordnungsrahmens waren in den wirtschaftswissenschaftlichen Diskursen des individualistischen Systems "gesetzt". In den deutschsprachigen Wirtschaftswissenschaften boten sich dabei die konstituierenden Prinzipien der Wettbewerbsordnung von Walter Eucken (1952) an, zur Referenznorm erhoben zu werden, nicht zuletzt weil sie jeder Ökonom als Student aus dem Pflicht-

\footnotetext{
2 Siehe auch die von Paul Hensel begründete Schriftenreihe zum Vergleich wirtschaftlicher Lenkungssysteme.
} 
fach Wirtschaftspolitik oder aus der Literatur kannte. Diese nicht „werturteilsfreie“ Grundlage der westdeutschen Wirtschaftswissenschaften wurde von ostdeutschen Ökonomen kritisiert, indem sie ihren Studenten den „apologetischen Charakter“ des Ordoliberalismus aufzuzeigen versuchten und deren Anhänger als „Vulgärökonomen“ verurteilten, die allein auf die Sicherung des „Sondereigentums“ abstellten (Turley 1961: 12). Weil diese Kritik ebenfalls nicht ohne eine ideologische Rückbindung der Forschungsperspektive auskam, drängte sich eine kritische Auseinandersetzung mit der Werturteilsfreiheit in den Wirtschaftswissenschaften nicht unmittelbar auf.

Spätestens nach dem Zerfall der sozialistischen Wirtschaftssysteme schienen immer mehr Ökonomen von normativen Ansätzen in der Wirtschaftspolitik Abstand zu nehmen, obwohl man aus der Retrospektive das genaue Gegenteil hätte erwarten können (Fukuyama 1989). Dieser Wandel hatte mehrere Gründe. Erstens hatte die einflussreiche Nachfolgegeneration der „Ordoliberalen“ ihren Wirkungszenit in Staat, Wirtschaft und Wissenschaft überschritten. Zweitens hatte die „Krise der keynesianischen Ökonomik" (Hicks 1974) Mitte der 1970er Jahre bereits einen methodischen Paradigmenwechsel eingeleitet, so dass insbesondere empirische Beiträge in der Fachliteratur immer stärker vertreten waren. Darüber hinaus hatte sich, drittens, eine neue Debatte um das Werturteilsproblem in den Sozial- und Wirtschaftswissenschaften entzündet. So fiel der gesellschaftliche Umbruch mit einem Generations- und Methodenwechsel zusammen, so dass sich eine Reflexion über die zukünftige Ausrichtung und Methodik der traditionellen Ordnungsökonomik anbot.

Lediglich im Hinblick auf ihren schwindenden Einfluss fand eine Auseinandersetzung um die Ordnungsökonomik in Deutschland seit Mitte der 1980er Jahre statt. Die zunehmende Verschuldung und die Persistenz der Arbeitslosigkeit hatten zu einer kontrovers geführten Debatte um die Zukunftsfähigkeit der Sozialen Marktwirtschaft geführt, die mehr zur inhaltlichen Wiederbelebung als zur Neuausrichtung der traditionellen Ordnungsökonomik führte: Es wurden die ,immer deutlicher gewordenen Defekte der interventionistischen Wirtschaftspolitik“" [der 1960er und 1970er Jahre, Anm. der Autoren] diskutiert, die das „Ordnungsdenken“ zusehends ,überlagert“ hatte (Cassel et al. 1988: 7). Diese „Renaissance der Ordnungspolitik“ konservierte das ordoliberal-normative Paradigma der Wirtschaftspolitik bis weit in die 1990er Jahre.

In dieser Diskussion wurde jedoch weder eine methodische Reflexion angelegt, noch der Weiterentwicklung und Modernisierung des traditionellen Ansatzes der Ordnungsökonomik nähere Beachtung geschenkt: Beiträge, die zum Beispiel die ordnungspolitischen Argumentationen hinsichtlich ihres „kryptonormativen“ Gehaltes erörterten und die Herangehensweise der Ordoliberalen kritisierten (u. a. Frey 1981; Kirchgässner 1988) oder eine Anbindung an die Institutionen- und Konstitutionenökonomik versuchten (Leipold 1988; Hoppmann 1987), fanden auf diese Weise nur eingeschränkt Eingang in die Forschungsprogrammatik der damaligen Ordnungsökonomik. Hinter der Kritik standen Anhänger der erstarkenden Forschungsrichtung der Neuen Politischen Ökonomie. Neue Perspektiven zur Weiterentwicklung des ordnungsökonomischen Forschungsansatzes lieferten einerseits die Public Choice Theorie und die Verfassungsökonomik (Buchanan 1984, 1987) und andererseits die Neue Institutionenökonomik (Richter 1987, 1988). 
Vorübergehend bleibt festzuhalten, dass diese Kritik an der Ordnungsökonomik sowie deren Weiterentwicklungsversuche von der vermeintlichen „Renaissance der Ordnungspolitik" überlagert und im Zuge des Generationswechsels nicht wahrgenommen wurde. Dieser Umstand verzögerte die Modernisierung der Ordnungsökonomik erheblich und könnte ein Grund dafür sein, warum der jüngste Methodenstreit in der bekannten Art und Weise ausufern konnte. Die Notwendigkeit zur methodischen Reflexion in der Ordnungsökonomik wurde jedoch bereits Ende der 1980er Jahre offen gelegt.

\subsection{Die Kritik der 1980er Jahre an der traditionellen Ordnungsökonomik}

In seinem Beitrag zur Festschrift für Artur Woll kritisiert Kirchgässner (1988) die Ordnungsökonomik aus der Perspektive der Neuen Politischen Ökonomie. ${ }^{3}$ In der Ordnungsökonomik der Freiburger Schule wird zwischen Ordnungstheorie und Ordnungspolitik unterschieden (Vanberg 1997: 50). Die Ordnungstheorie stellt das „theoretische Paradigma“" der wissenschaftlichen Arbeit der Freiburger Schule dar (Vanberg 2004: 9) und befasst sich mit der „Analyse und Erklärung der Funktionseigenschaften alternativer Rechts- oder Regelordnungen" (Vanberg 1997: 49). Im Zentrum dieser Analyse steht die Frage, wie unterschiedliche Regelordnungen auf die jeweilige Wirtschaftsordnung wirken. Die einer Wirtschaftsverfassung zugrunde liegenden Regeln werden in der Ordnungstheorie auf ihre Wirkung hin untersucht (Streit 1996: 815). Die Ordnungspolitik hingegen bildet das „politische Paradigma“ der Ordnungsökonomik der Freiburger Schule. Dabei wird die Frage aufgeworfen, wie die aktuelle Wirtschaftsordnung durch wirksame Reformen bzw. Regeländerungen verbessert werden kann (Vanberg 2004: 9). Aus dem ordnungstheoretischen Erkenntnisstand wird ordnungspolitisches Wissen gewonnen, um zur Lösung praktischer Ordnungsprobleme beizutragen. Das wissenschaftliche Vorgehen der Ordoliberalen ist somit nicht auf die Analyse existierender Ordnungen beschränkt, sondern versucht zudem, die Charakteristika einer wünschenswerten Ordnung zu erarbeiten (Vanberg 1997: 49f.). In der traditionellen ordnungsökonomischen Perspektive ist das normative Referenzkriterium insbesondere in der Konzeption der Wettbewerbsordnung von Eucken zu verorten (Eucken 1952). Vorübergehend löst sich seine Nachfolgegeneration zwar von diesem Kriterium während der Hochphase des Keynesianismus (Böhm 1973: 42; Lenel 1971). Die „Ordnungspolitische Renaissance“ der 1980er Jahre rückt sie aber wieder in die Nähe der konstituierenden Prinzipien. Die moderne Ordnungsökonomik bzw. die Verfassungsökonomik verzichten auf eine solche Rückbindung an ein positives Programm, indem dort die normativ-individualistische Perspektive auf das Legitimationsprinzip ausgedehnt wird, so dass die allgemeine Zustimmung bzw. eine Einstimmigkeitsregel an die Stelle eines ordnungspolitischen Dekalogs rückt. In diesem Punkt emanzipiert sich die Verfassungsökonomik von der traditionellen ord-

3 Er bezieht diese Kritik auf die „Ordnungstheorie“, die er jedoch nicht inhaltlich, sondern über jene Autoren definiert, die im ORDO Jahrbuch publizieren (Kirchgässner 1988: 54, Fn. 3). Damit zielt seine Kritik auf eine heterogene Gruppe im Kreis der deutschsprachigen Wirtschaftswissenschaftler ab, zu denen nicht nur die Gründer der Freiburger Schule zählen, sondern denen vielmehr deren Nachfolger und weitere „Schulen“ der Ordnungsökonomik hinzuzurechnen sind. 
nungsökonomischen Diskussion mit der Forderung, den Bürger als Souverän des Gemeinwesens anzuerkennen (Vanberg 1988).

Kirchgässners Kritik setzt insbesondere am normativen Anliegen der traditionellen Ordnungsökonomik an. Wenngleich viele Beiträge vordergründig werturteilsfrei gehalten seien, sei ihnen eine „kryptonormative“ Argumentation zu Eigen, da wesentliche Argumente nicht als Werturteile gekennzeichnet seien, sie aber dennoch wertenden Charakter hätten (Kirchgässner 1988: 62ff.). Wenn Werturteile in ordnungspolitischen Argumentationen so selbstverständlich verwendet würden, erwecke dies den Eindruck, dass hier ein überlegenes Wissen zur Verfügung stehe (Kirchgässner 1988: 53). Letztlich lehne sich die Ordnungsökonomik damit an die Figur des wohlmeinenden Diktators der Wohlfahrtsökonomik an und vernachlässige die theoretische Analyse politischer Prozesse. Die Ordnungsökonomik bleibt dadurch hinter dem Erkenntnisstand der Neuen Politischen Ökonomik zurück, was dazu führt, dass Vertreter der Ordnungspolitik Handlungsempfehlungen als wissenschaftlich begründet ansehen, obwohl sie ihre individuellen Werthaltungen reflektieren. ${ }^{4}$ Eucken selbst lehnte eine Ausdehnung ökonomischer Analyse auf den Datenkranz, zu dem die rechtlichen und politischen Rahmenbedingungen gehören, explizit ab (Eucken 1940: 156ff.). Ebenso kritisch sieht Böhm (1957) eine ordnungstheoretische Analyse politischer Entscheidungsprozesse.

Den Anhängern der traditionellen Ordnungsökonomik ging es während der Renaissance der Ordnungspolitik in den 1980er und 1990er Jahre vor allem darum, die Ordnungsgrundsätze Walter Euckens zu kanonisieren, um ihre Gegenposition zum Interventionismus zu legitimieren. ${ }^{5}$ Sie setzten sich dadurch dem Vorwurf aus, ideologisch ausgerichtet zu sein. Derart „dogmatische Diskussionen“ kenne man nur aus der „Theologie, wo sie sicherlich ihren Platz haben“ (Kirchgässner 1988: 64). Dies „spreche $[\ldots]$ für dessen Begründer, Eucken, aber nicht unbedingt für seine Nachfahren. Es könnte eher ein Indiz dafür sein, dass eine Weiterentwicklung dieser Theorie seit seinem Tode nicht mehr stattgefunden hat" (Kirchgässner 1988: 65). Dadurch tendierten Ordnungsökonomen dazu, in den „Ruf nach dem starken Mann“ einzustimmen, der den „Mut zu einer langfristig angelegten Politik“ aufbringt, um das ordnungspolitische Programm umzusetzen (Kirchgässner 1988: 59).

4 Giersch weist hingegen darauf hin, dass Ökonomen, wenn sie wirtschaftspolitische Handlungsempfehlungen unterbreiten, ihre eigenen Wertvorstellungen explizit benennen sollten. Siehe dazu Giersch (1993), noch deutlicher: „Für den Diskurs ist es nach meinem Dafürhalten notwendig, dass man andere über die mögliche Wertbezogenheit des eigenen Urteils nicht hinwegtäuscht, also nicht mogelt. Aber noch setzt man sich dem Ideologieverdacht aus, wenn man offen erklärt, von welchem Standpunkt im Wertesystem die alternativen wirtschaftlichen Perspektiven, die man aufzeigt, gesehen wurden und zu sehen sind. Vieles, was sich als objektive Wissenschaft präsentiert, hat subjektive Komponenten“" (Giersch 1994: 244).

$5 \quad$ Ein Blick in die Beiträge des ORDO Jahrbuches stützt diese These. Dabei fällt ebenso auf, dass die Debatte um die „Renaissance der Ordnungspolitik“ bereits Mitte der 1980er Jahre die wenigen Beiträge überlagert, die sich mit der Analyse politischer Prozesse beschäftigen. In Band 34 von ORDO finden sich mehrere Beiträge zu diesem Thema, die sich allerdings für eine Beschränkung der Demokratie aussprechen (Rowley 1982: 51; Molitor 1982: 33ff.). 
Wenngleich ein totalitäres Staatsideal für Eucken und seine Mitstreiter in den Widerstandskreisen nicht geltend gemacht werden kann (Dathe 2010) und Eucken „erklärter Gegner jeder Vorstellung unbegrenzter Regierungsmacht“ sowie „unbegrenzter Demokratie" war (Streit und Wohlgemuth 2000: 467), wird hier ein gravierendes methodologisches Problem offen gelegt, das nur unzureichend von traditionellen Ordnungsökonomen erkannt und bearbeitet wurde. ${ }^{6}$ Zur Modernisierung fordert Kirchgässner in Anlehnung an Popper die traditionelle Ordnungsökonomik auf, die Frage nach der institutionellen Ausgestaltung des Staates anhand des folgenden Leitgedankens zu beantworten: „Wie können wir politische Institutionen so organisieren, dass es schlechten oder inkompetenten Herrschern unmöglich ist, allzu große Schäden anzurichten" (Popper 1957: 145), und schlägt dazu vor, mehr direkte Einflussmöglichkeiten für die Bürger zu schaffen (Kirchgässner 1988: 68). Institutionen „der direkten Demokratie“ könnten ein „erhebliche[s] Gegengewicht gegen den Einfluss von Interessengruppen auf die Politik bilden" und so die positiven Ordnungsvorstellungen der Freiburger in einem Abstimmungstest legitimieren (Kirchgässner 1988: 68). Diese Argumente sind im Sinne der Verfassungsökonomik, da die Bürger in ihrer legitimatorischen Funktion institutionell berücksichtigt werden: „Gerade die Stärkung und nicht die Verringerung der demokratischen Rechte könnte somit zu Ergebnissen führen, wie sie von den Vertretern der Ordnungstheorie gewünscht werden" (Kirchgässner 1988: 69). ${ }^{7}$

\subsection{Die Weiterentwicklung des Freiburger Ansatz von Viktor Vanberg}

In mehreren Beiträgen verknüpft Vanberg $(1988,1997)$ den traditionellen Forschungsansatz der Freiburger Schule mit dem Forschungsprogramm der Verfassungsökonomik. Im Zentrum des verfassungsökonomischen Forschungsansatzes steht, wie sich die Wahl einer Handlungsrestriktion im Sinne einer Entscheidung über Verfassungsregeln, auf die korrespondierende Handelnsordnung auswirkt (Vanberg 2004a: 3). Die Gesamtheit aller Regeln, die in einer Gesellschaft gelten, wird von Buchanan (1975) auf der konstitutionellen Ebene und von Hayek $(1960,1967)$ in der Regelordnung verortet. Die korrespondierende Handlungsebene befasst sich mit den individu-

6 Hinsichtlich der Freiburger Schule sollte der historische Kontext berücksichtigt werden: Zwischen 1933 und 1949 waren die im Reich verbliebenen Freiburger einem mehr oder weniger starken Zensurapparat unterworfen. Zur Weiterentwicklung der pluralistischen Grundordnung beizutragen und gleichzeitig innerhalb eines Regimes zu leben, das diktatorisch bzw. von einer Militäradministration regiert wird, kann kaum eingefordert werden. Vor der Zensur hatte Eucken vor einem wohlmeinenden, diktatorischen Staatsideal gewarnt, das „heute“ mittels eines „Glaube[ns] an den Staat und zwar an den totalen, alles beherrschenden Staat“ zu einem „Religionsersatz“ geworden sei (Eucken 1932: 306). In diesem „,totalen Staat der Zukunft wird heute von vielen Deutschen ein übermenschliches alles vermögendes Wesen gesehen“ (Eucken 1932: 306). Gegen diese radikale Staatsauffassung setzte Eucken das Konzept des „,starken Staates“, um „den einzelnen Menschen vor der Übermacht des Staates schützen“ (Eucken 1952: 337).

$7 \quad$ Er nimmt auf der damaligen schmalen Basis empirischer Studien vorweg, was sich später als empirisch fundiert herausstellte. Siehe dazu Kirchgässner, Feld und Savioz (1999) und Feld und Kirchgässner (2009). 
ellen Entscheidungen oder mit den Ergebnismustern, die sich unter dem auf der konstitutionellen Ebene gewählten Regelrahmen ergeben. ${ }^{8}$

Der Forschungsgegenstand der Verfassungsökonomik besteht somit in der Analyse der Funktionseigenschaften unterschiedlicher Regelordnungen bzw. Verfassungsregeln im Hinblick auf die resultierenden gesellschaftlichen Interaktionen der Individuen. Die Verfassungsökonomik belässt es jedoch nicht bei der Analyse der Regelordnung oder Institutionen, sondern fragt vielmehr danach, wie diese gestaltet sein sollten, um dem Interesse der betroffenen Individuen zu dienen (Vanberg 1998: 70). Die Frage, welche Regeln wünschenswert sind, beantwortet die Verfassungsökonomik nicht anhand von externen Werturteilen, sondern anhand des Kriteriums der freiwilligen Zustimmung, das als Bewertungsmaßstab einer Regelwahl auf allen Entscheidungsebenen anzuwenden ist (Vanberg 1997: 713).

Eine solche Vorgehensweise ist jedoch nur auf der Grundlage geeigneter Verhaltensannahmen sinnvoll. Die Verfassungsökonomik beruht auf der Annahme des methodologischen Individualismus, nach dem ausschließlich das Individuum als handelnde Einheit anzusehen ist. Kollektive handeln nicht autonom. Eine organische Sicht von (staatlichen oder privaten) Organisationen lehnt die Verfassungsökonomik ab. Somit entscheiden allein die Individuen gemäß ihren Präferenzen und Zielvorstellungen über eine aus der Regelwahl resultierende Handelnsordnung darüber, welche Regel oder Verfassung angewendet werden sollte. Die Interessen der Individuen werden somit zum „Legitimationsprinzip“ einer Regelwahl. Damit folgt die Verfassungsökonomik als angewandte Wissenschaft einem normativ verstandenen Individualismus (Vanberg 2004a: 4), gemäß dem die Beurteilung einer Regelwahl nur bei den betroffenen Personen selbst liegt, weil nur das Individuum Quelle eines Werturteils ist. ${ }^{9}$ In dieser Hinsicht stellen die Präferenzen der Menschen im Hinblick darauf, unter welcher Regelordnung sie leben möchten, das konstitutionelle Interesse dar. Aus dem vertragstheoretischen Paradigma der Verfassungsökonomik folgt zudem, dass die Individuen nicht nur freiwillig, sondern auch (quasi-) einstimmig der Regelreform zustimmen müssen. ${ }^{10}$ Eine freiwillige Vereinbarung über Regeln bzw. deren Änderungen ist nur dort zu erwarten, wo konsensfähige konstitutionelle Interessen bestehen (Vanberg 1997: 56). Somit folgt aus der vertragstheoretischen Perspektive der Verfassungsökonomik, dass eine wünschenswerte Verfassung bzw. eine Regelwahl nur dann zu legitimieren ist, wenn diesbezüglich erstens die betroffenen Individuen ein konsensfähiges konstitutionelles Interesse aufweisen und zweitens auf dieser Grundlage eine freiwillige $\mathrm{Zu}$ stimmung ermöglicht wird.

8 Die Handelnsordnung wird von Buchanan dabei als nachkonstitutionelle Ebene bezeichnet (siehe Vanberg 1998: 73).

9 Diese zentrale normative Annahme bildet auch das Fundament der Buchanan'schen Vertragstheorie: „The critical normative presupposition on which the whole contractarian construction stands or falls is the location of value exclusively in the individual human being " (Brennan/Buchanan 1985: 25).

10 Zur Unterscheidung zwischen faktischer und freiwilliger Zustimmung, wobei allein auf Letztere aus verfassungsökonomischer Perspektive abgestellt wird, siehe Vanberg (1994: 208ff.). 
Vor diesem Hintergrund ist das Ziel, die Handlungsarenen des Marktes und des Staates zu verbessern, mit der Suche nach geeigneten Arrangements zur Wahrung konstitutioneller Interessen verbunden. Ein dazu korrespondierendes Vorgehen stellt die Gewährleistung der Konsumenten- und Bürgerinteressen als entscheidende RegelgröBe auf der Verfassungsebene dar:

"Improving the legal-institutional framework [...] this means for markets to adopt and to maintain an economic constitution that enhances consumer sovereignty, and for the political arena it means to adopt and to maintain constitutional rules that enhance citizen sovereignty" (Vanberg 2004a: 26).

Die Konzepte der Konsumenten- und Bürgersouveränität bilden verfahrensorientierte Steuerungsideale für die Regelebene, die in ihrer konstitutionellen Verwirklichung den Wirkungsmittelpunkt auf der nachkonstitutionellen Ebene in der Befriedigung der konstitutionellen Interessen der beteiligten Personen finden. Anders gewendet: Diese Konzepte befriedigen als Steuerungsideale die konstitutionellen Interessen der Betroffenen umso mehr, je weitreichender sie in der Regelordnung Anwendung finden. Werden Bürgerinteressen in der Regelordnung des politischen Systems einer Gesellschaft in hohem Maße berücksichtigt, so haben die betroffenen Individuen an einer solchen Verfassung ein konsensfähiges konstitutionelles Interesse. Dabei geht die Bürgersouveränität direkt aus der Annahme des normativen Individualismus hervor, da die Bürgerinteressen die einzige Legitimationsquelle für Entscheidungen in der politischen Handlungsarena darstellen (Vanberg 2004a: 21).

Analog zu dieser Herangehensweise liefert das Ideal der Konsumentensouveränität das Kriterium, an dem existierende Wirtschaftsordnungen daraufhin untersucht werden können, inwiefern sie den Interessen der Individuen als Konsumenten folgen. Mit diesem Konzept ist die Suche nach „Verfahren oder Spielregeln“ verbunden, die „die größtmögliche Gewähr dafür bieten, dass die Konsumentenwünsche die entscheidende Regelgröße im Wirtschaftsprozess“ darstellen (Vanberg 1997: 63). Eine dem Ideal der Konsumentensouveränität folgende Wirtschaftsverfassung zeichnet sich dadurch aus, dass in ihr die Konsumenteninteressen gegenüber denen der Produzenten Priorität zukommt, und die Konsumenteninteressen in letzter Instanz den Wirtschaftsprozess steuern (Vanberg 1997: 64). Aus verfassungsökonomischer Perspektive ist daher eine der Konsumentensouveränität folgende Wirtschaftsverfassung zu legitimieren, da für eine solche Verfassung ein allgemein zustimmungsfähiges Interesse besteht (Vanberg 2004a: 19).

Zentral ist die Erkenntnis, dass der von der Freiburger Schule angestrebte Leistungswettbewerb ein Steuerungsideal darstellt, das gleichermaßen verfahrensorientiert wie das Konzept der Konsumentensouveränität ist. Es zielt darauf ab, die Regeln auf der konstitutionellen Ebene so auszugestalten, dass „eine größtmögliche Reagibilität gegenüber den Konsumentenwünschen erreicht wird“ (Vanberg 1997: 63). Je umfassender dem Prinzip des Leistungswettbewerbs in einer Ordnung Rechnung getragen wird, desto mehr ist den konstitutionellen Interessen der Individuen gedient. Gleiches gilt für das ordoliberale Verfassungsideal der „privilegienfreien Ordnung von Rechtsgleichen". Mit dem Grundsatz, jedem Individuum die gleichen Rechte und den gleichen Status im Rahmen einer Person des Privatrechts zu verleihen, bewirkt dieses Ideal eine „verfassungspolitische Grundentscheidung zugunsten“ der Gesellschaft, das 
den „konsensfähigen konstitutionellen Interessen“ der Gesellschaftsmitglieder entspricht (Vanberg 1997: 57).

Wenngleich Eucken oder Böhm über das Konzept der konsensfähigen konstitutionellen Interessen in Unkenntnis waren, dürften beide ,vermutlich keine Schwierigkeiten gehabt haben“, es als Legimitationskriterium und ,als letztendlichen normativen Maßstab gelten zu lassen“ (Vanberg 1997: 65). Ihre Argumentation ist weder systematisch „,naturrechtlich“, oder im engeren Sinne - wie von Voigt (1997) vermutet - „,metaphysisch“ legitimiert (Vanberg 1997: 64). Auch wenn Bezüge zu normativen Vorstellungen hergestellt würden, seien sie keinesfalls ,systematischer“ Natur, oder gar „,konstitutiv für dieses [Forschungs-] Programm“ (Vanberg 1997: 56). Daher könne das Freiburger Forschungsprogramm ohne jeden Anspruch auf einen Sonderstatus als „normative Wissenschaft" ganz im Sinne des methodologischen Postulats der Werturteilsfreiheit betrieben werden.

Eine weitere Gemeinsamkeit der beiden Forschungsprogramme stellt die konstruktive Herangehensweise dar, die Ordnung des Gesellschaftswesens als eine Problemstellung der Verfassungs- bzw. Regelwahl zu verstehen (Vanberg 1988: 27). Zusammen mit dem Kriterium der freiwilligen Zustimmung bildet die konstruktive Herangehensweise die beiden Elemente der normativen Dimension der Verfassungsökonomik.

Das Konzept der Konsumentensouveränität kann damit als „Leitbild für die ordnungspolitische Gestaltung der marktlichen Wettbewerbsordnung“ interpretiert werden, so wie „das Leitbild der ordnungspolitischen Gestaltung des politischen Wettbewerbs“ mit dem Begriff der Bürgersouveränität umschrieben werden kann: „Wie eine solche Ordnungspolitik für den öffentlichen Bereich aussehen könnte, ist eine der zentralen Fragen, denen sich eine das Freiburger Forschungsprogramm weiterführende Ordnungsökonomik zu widmen hat" (Vanberg 1997: 65f.). Dieser Appell gilt vor allem der ,angewandten Ordnungsökonomik“, insbesondere der Neuen Politischen Ökonomie, die untersuchen soll, wie Institutionen im Sinne der Bürgersouveränität zur Erlangung der freiwilligen Zustimmung am ,angemessensten praktisch angewandt und operationalisiert" werden können (Vanberg 1997: 65). Vanberg gelingt somit das, was von Kirchgässner (1988) mit dem Verweis auf die fehlende Theorie politischer Entscheidungsprozesse und die normative Orientierung der Ordnungspolitik abgestritten wird: Die Integration der Ordnungsökonomik in das Forschungsprogramm der Verfassungsökonomik. ${ }^{11}$ Die Verfassungsökonomik und die „,traditionelle Ordnungsökonomik“ scheinen sich unversöhnlich gegenüber zu stehen. Ersetzt man jedoch das Referenzkriterium der konstituierenden Prinzipien der Wettbewerbsordnung durch das Prinzip der Zustimmungsfähigkeit löst sich dieser Konflikt auf.

11 Kirchgässner (1988: 70) stimmt nicht nur dem Legitimationskriterium der Verfassungsökonomik zu, sondern versteht es als konkreten Forschungsauftrag (1988: 68), da er Institutionen dahingehend untersuchen möchte, ob ihre objektivierbaren Funktionseigenschaften eine hohe Reagibilität auf Bürgerinteressen besitzen. Dabei werden Arrangements empfohlen, die eine bestmögliche Ausgestaltung unter dem doppelten Ziel der institutionellen Wirksamkeit und der Reagibilität auf die Bürgerinteressen gewährleisten. Dieses gemeinsame Ziel geht aus einem normativ-individualistischen Erkenntnisprogramm hervor. 


\subsection{Die Positionen deutscher Ökonomen im „neuen Methodenstreit“}

Der „neue Methodenstreit“ (Caspari/Schefold 2011: 11) oder „Ökonomenstreit“ (Bräuninger et al. 2011: 24) entzündete sich im Jahr 2009 an der Frage der Lehrstuhlbesetzung und -ausrichtung der Wirtschafts- und Sozialwissenschaftlichen Fakultät der Universität zu Köln. Während eine Seite die Umwidmung wirtschaftspolitischer und finanzwissenschaftlicher Lehrstühle kritisierte und einen von 83 Professoren unterschriebenen Aufruf mit dem Titel „Rettet die Wirtschaftspolitik an den Universitäten!“ initiierte, verteidigte die andere Seite die methodische Ausrichtung der modernen Volkswirtschaftslehre, kritisierte die Ordnungsökonomik und antwortete mit einem von 188 Professoren unterschriebenen Aufruf mit dem Titel „Baut die deutsche VWL nach internationalen Standards um!“".12 Neben der Mathematisierung der Volkswirtschaftslehre und dem Vorwurf einer unzureichenden Berücksichtigung von Institutionen argumentierte Hans Willgerodt (2009), der zusammen mit Christian Watrin die Diskussion um die Kölner Berufungspolitik begonnen hatte, zudem ganz explizit gegen das Wertfreiheitspostulat in der modernen Ökonomik. Bachmann und Uhlig (2009) antworteten darauf lediglich mit Bezügen zu den verwendeten Methoden und zur internationalen Wettbewerbsfähigkeit der deutschen Volkswirtschaftslehre. Daraus entwickelte sich eine sehr interessante und aufschlussreiche Debatte um die Zukunftsfähigkeit der Ordnungsökonomik.

Goldschmidt, Wegner, Wohlgemuth und Zweynert (2009) stellen insbesondere auf die gesellschaftliche Verantwortung der Ökonomen ab. Die Wirtschaftswissenschaften hätten sich „seit ihrer Begründung durch Adam Smith“ mit zwei Problemen „befasst“: „Erstens mit den Gesetzmäßigkeiten von wirtschaftlichen Prozessen; zweitens mit den Wechselwirkungen dieser Prozesse mit der sozialen Umwelt" (Goldschmidt et al. 2009). „Ordnungsökonomisches Denken“ hinge nicht an dem Begriff, sondern an der Art und Weise, wie man sich den Problemen der modernen Gesellschaft zuwende. In jedem Falle müsse die Ordnungsökonomik beiden Fragestellungen - „der nach den innersystemischen Eigenschaften des Wirtschaftsprozesses und der nach dem gesellschaftlichen Bezug des Wirtschaftens - gerecht" werden (Goldschmidt et al. 2009). Daher fordern sie die konsequente Berücksichtigung der „Interdependenz der Ordnung“ im Sinne einer „kontextualen Analyse des Wirtschaftens“. Wenn sich die Wirtschaftswissenschaft von der Ordnungsökonomik verabschiede, bestünde die Gefahr, dass sie ihre gesellschaftliche Relevanz verliere.

Zur Neuausrichtung der Wirtschaftswissenschaften schlagen sie drei Strategien vor, die gemeinsam darauf abzielen, Probleme der ,gesellschaftliche[n] Realität" besser erkennen und lösen zu können: Erstens, durch die Wiederanbindung der Wirtschaftswissenschaften an die Sozialwissenschaften, indem sie auf die Interdependenz der Ordnungen verweisen. Zweitens drängen sie darauf, dass die Wirtschaftswissenschaften ihre kulturökonomische Perspektive wiederentdeckten, und drittens, dass sie die Interdisziplinarität zu anderen Wissenschaften suchen sollen. Mit diesem sozialwissenschaftlich-holistischen Ansatz versuchen sie, die Ordnungsökonomik innerhalb der zudem insbesondere die Beiträge von Rüdiger Bachmann und Roland Vaubel in diesem Band sowie Plickert (2009). Lars Feld hat keinen der beiden Aufrufe unterschrieben. 
Wirtschaftswissenschaften anschlussfähig zu machen, ohne dabei auf die „alten Denker“ Rekurs nehmen zu müssen. In dieser Ausrichtung sei die Ordnungsökonomik „kein deutscher Sonderweg“, insbesondere wenn man ihn nicht als „dogmenhistorische Episode“ betrachte, sondern als „modernes, interdisziplinär wie international anschlussfähiges Forschungsprogramm."

Haucap (2009) bemängelt, dass der Methodenstreit auf das Thema „formale Methode versus qualitative, verbale Analyse“ reduziert werde. Zentraler wären die beiden Fragestellungen, wie - erstens - der Bürger von der wirtschaftswissenschaftlichen Forschung profitiere, wenn er sie schon bezahle (Haucap 2009: 20), und - zweitens - wie die „richtige Mischung“ zwischen anwendungsorientierter Forschung und Grundlagenforschung zu finden sei. Mit dieser politökonomischen Perspektive schließt er implizit an die Giersch'sche Bringschuld an und wirft gleichzeitig ein legitimatorisches Problem der Wissenschaften auf. Zur Neuausrichtung der Wirtschaftswissenschaften führt er an, dass exzellente Ökonomen bestmöglich alle Disziplinen beherrschen sollten: „Theorie, Empirie und institutionelle Kenntnisse“. Da jedoch nicht alle „kleine Ronaldos“ der VWL seien und „,nicht alle Krugman und Stiglitz“ hießen, ist eine Arbeitsteilung zwischen „sich ergänzenden Spezialisierungen“, aber keine Monokultur eines Lagers sinnvoll.

Schmidt und aus dem Moore (2009) finden es „äußerst bedenklich“, dass „Themen wie individuelle Freiheit, individuelle Verantwortung im politischen Prozess und Bekenntnis zu einer marktwirtschaftlichen Ordnung im Bewusstsein der Bevölkerung an Bedeutung verloren haben“. Gleichzeitig halten sie aber auch am Dreiklang aus Mikroökonomik, Makroökonomik und Ökonometrie fest. Sie verteidigen die „Formalisierung" mit dem Argument, dass die quantitativ orientierte Forschung keineswegs blind für institutionelle Details sei. Sie sehen den Methodenstreit in dieser Richtung „völlig ins Leere“ laufen: Schließlich sei „das Denken in großen Zusammenhängen [...] in der Tat nach wie vor eine der größten Herausforderungen für die Disziplin, denn einzelwirtschaftliche Analyse allein“ könne „die Fragen nach dem besten ordnungspolitischen Rahmen und nach der angemessenen Form staatlicher Eingriffe ins Wirtschaftsgeschehen nicht ersetzen.“ Diese „Aufgabe“ ließe „sich jedoch nicht dadurch meistern, dass man auf die Vorzüge“ verzichte, „die aus der in den vergangen Jahrzehnten erfolgten Mathematisierung des Fachs zu schöpfen“" waren. Vielmehr zwinge die Mathematik zur konsistenten Argumentation und „wissenschaftlichen Bescheidenheit", besonders in der empirischen Forschung. Ökonometrie sei deshalb notwendig, weil Ökonomen empirische „Belege für und wider ihre theoretischen Argumente“ benötigten, die selten ,im Labor" zu sammeln seien. Schließlich gebe es keine sinnvolle Alternative für „Gesellschaften, die dem Erbe der Aufklärung folgen und sich bei Entscheidungen nicht an religiöse Dogmen oder ideologischen Vorhaben orientieren“, als auf Basis einer „evidenzbasierte[n] Politik“ nach Lösungen für reale Probleme zu suchen. Es müsse dabei der Grundsatz in der theoretischen und der empirischen Analyse gewahrt bleiben, dass „,so einfach wie möglich und so komplex wie nötig“ vorzugehen sei. Daher sei auch der „entscheidende Gradmesser für gute Forschung“ darin zu finden, ,wie gut sie die inhaltliche Fragestellung adressiert“ und nicht in der Frage, „ob ein Forscher komplexe Methoden anwenden“ könne. 
Sinn (2009) kritisiert in seinem Beitrag zum Methodenstreit, dass die Institutionenökonomik in den Wirtschaftswissenschaften „zu kurz" gekommen sei. Auch in den besten „internationalen Zeitschriften unseres Faches“ sei dies zu beobachten. Die Volkswirtschaftslehre benötige „heute wieder mehr Ökonomen, die bereit sind, sich mit den Details des staatlichen Ordnungsrahmens und der Funktionsweise des Staatsapparates zu beschäftigen“. Er begründet diese Forderung mit dem Argument, dass der Staat in den westlichen Ländern „mehr als die Hälfte des Volkseinkommens für seine Zwecke“ absorbiere und so „massiv in die Entscheidungsfreiheit der Bürger eingreife“. Daher sei es notwendig, insbesondere die „Gesetze und Verordnungen“ zu berücksichtigen, die „das wirkliche Geschehen in der Ökonomi“" beeinflussten. Dazu gehörten auch die staatlichen Institutionen. Ebenso sei die Zukunft der Finanzwissenschaft in den deutschen Wirtschaftswissenschaften bedroht, weshalb er sie in seinem Reformaufruf ebenfalls mit einbezieht. Die Wirtschaftswissenschaft sei in „einem gleichgewichtigen Dreiklang von Theorie, Institutionenlehre und Ökonometrie“ zu intonieren, um der Wirtschaftspolitik „mit fundierten Empfehlungen dienen zu können".

Kirchgässner (2009) vergleicht den Beitrag der 83 Professoren mit einem Ruf nach einem „deutschen Sonderweg“" den er grundsätzlich ablehnt: Wenngleich es hinsichtlich der methodischen Kritik an der quantitativen Ökonomik unbestritten sei, dass es „Arbeiten gebe, deren Bedeutung für die Lösung realer Probleme durchaus zweifelhaft" sind, seien „viele der rein verbalen Analysen, die sich zum Beispiel in deutschsprachigen Zeitschriften finden, stark ideologiegetränkt". Rückblickend auf seine frühere Kritik an der traditionellen Ordnungsökonomik ist es wenig überraschend, wenn Kirchgässner erneut die Notwendigkeit „partielle[r] mathematische[r] Analysen“ einfordert, obwohl sie nur bestimmte „Aspekte der Wirklichkeit betrachteten und andere ausblendeten, da „holistische Ansprüche“ letztlich nicht „einlösbar" seien. Hierbei richtet er sich gegen Ökonomen, die sich eine „Renaissance des Ordoliberalismus" wünschen. Wer Institutionenökonomik einfordere, und dieses Ziel mit dem Ordoliberalismus verbinde, sollte sich darüber im Klaren sein, dass jene dort behandelten Fragen „im Rahmen der modernen Institutionenökonomik“ bereits ausführlich diskutiert werden. So sei es weniger wichtig, dass ein Ansatz „allumfassend“ sei, sondern „dass er die für die Forschungsfrage relevanten Aspekte der Wirklichkeit hinreichend konkret“ abbilde. Dabei sei insbesondere „bei der Überprüfung von Hypothesen an der Wirklichkeit" Mathematik sehr hilfreich. Die „übertriebene" Abstraktion und die Formalisierung lässt er als Gegenargumente nicht gelten: Schließlich muss ,,jede realwissenschaftliche Analyse, die etwas über die Wirklichkeit aussagen will, [...] notwendigerweise von den meisten Aspekten der Wirklichkeit abstrahieren und sich einige wenige genauer vornehmen“. Für die zukünftige Ausrichtung der Wirtschaftswissenschaften sieht er Institutionen als wichtigste Beobachtungsgröße, die es mit Hilfe mathematisch-statistischer Methoden auf ihre Funktion hin zu überprüfen gilt. Ebenfalls fordert er eine konsequente Berücksichtigung des Weber'schen Wertfreiheitsprinzips. Daher ,dürfte es kaum negativ zu beurteilen sein, wenn in der Diskussion auch mathematische Modelle sowie moderne ökonometrische Verfahren zum Einsatz gelangen“. Die deutschen Wirtschaftswissenschaften dürften, sofern sie „weiterhin international eine Rolle spielen“ wollen, „sich nicht ins Schneckenhaus zurückziehen". 
Vanberg (2009) verortet den eigentlichen Kern des jüngsten Methodenstreits in der Frage, „worauf der wissenschaftliche Anspruch des Fachs zu gründen“ sei. In den Wirtschaftswissenschaften herrsche die Vorstellung vor, dass „die Wissenschaftlichkeit ökonomischer Beiträge [...] an den darin zur Anwendung kommenden mathematischen und statistischen Fertigkeiten zu messen" sei. Allerdings macht Vanberg seiner „Disziplin“ nicht den „Vorwurf“, dass sie sich der formalen Sprache der Mathematik bediene. Auch sehe er kein „Makel darin, dass sie statistische Verfahren“ nutze. Seine Kritik beginnt dort, wo der Forschungsehrgeiz „die Verfügbarkeit verwertbarer Daten zum Kompass dafür macht, welche Zusammenhänge man“ untersuche. Ökonomen gleich welcher Provenienz - sollten sich zudem stets darüber im Klaren sein, dass man es mit einem offenen, evolvierenden System zu tun habe, bei dem die „Komplexität der Bedingungsfaktoren“ sowie die vielfältigen „Wechselwirkungen erfinderischer menschlicher Handlungen“, den Möglichkeiten, „präzise quantitative Aussage zu treffen, enge Grenzen setzt." Vanberg mahnt hier zur Zurückhaltung bei der Verwertung der produzierten quantitativen Ergebnisse, die nicht zur Vorhersage von „konkreten Auswirkungen spezifischer Eingriffe“ herangezogen werden sollten, da das begrenzte Wissen der Wissenschaftler allein Mustervoraussagen zulasse und lediglich „typische Auswirkungen“ von „systematischen Änderungen in den Rahmenbedingungen“ erörtert werden. Die Ordnungsökonomik verfolge ein solches Forschungsprogramm der Mustervoraussagen. Allerdings sollte man dort, wo eine „quantitative Ausrichtung den Erkenntniszweck fördert“" sie „tunlichst nutzen“.

\subsection{Die Kritik an der Ordnungsökonomik im Lichte des neueren Methodenstreits}

Der neuere Methodenstreit in der deutschen Volkswirtschaftslehre war längst überfällig, weil die Perspektiven zur Weiterentwicklung der Ordnungsökonomik - wie zum Beispiel die Überführung des traditionellen Forschungsprogramms der Freiburger Schule in die Verfassungsökonomik - nicht hinreichend wahrgenommen worden sind. Die fehlende Neuorientierung lässt sich einerseits durch die „Renaissance der Ordnungspolitik“ in den 1980er Jahren, welche die Vertreter der „traditionellen“ Ordnungsökonomik in Sicherheit wiegte, und andererseits durch den Generationswechsel in den Wirtschaftswissenschaften während der 1990er Jahre erklären, der gleichsam einen methodischen Bruch in Lehre und Forschung zur Folge hatte. Die methodische Fortentwicklung der Ökonomik ging an der „traditionellen“ Ordnungsökonomik vielfach vorbei. Ihr Potential wurde daher für die Ordnungsökonomik kaum erschlossen.

Analysiert man die Hauptaussagen dieses Methodenstreits auf die Frage hin, was die Ordnungsökonomik zukünftig zu leisten hat, stellt man fest, dass die Kritik der 1980er Jahre sowie die Weiterentwicklung der Ordnungsökonomik hin zur Verfassungsökonomik über das hinausgehen, was im jüngsten Methodenstreit geliefert wurde. Es wird wohl unbeantwortet bleiben, wie der jüngste Methodenstreit so losgelöst von dieser Debatte geführt werden konnte, deren gemeinsamer Nenner allenfalls in der notwendigen Berücksichtigung von Institutionen zu finden ist. Vermutlich liegt dies daran, dass sich die Ordnungsökonomen selbst mit dieser Diskussion nicht beschäftigt haben. Allein die Tatsache, dass die kritischen Beiträge einen Ideologisierungs- bzw. 
Formalisierungsverdacht auf der Gegenseite anmahnen, belegt, dass die Anhänger beider Seiten den bereits stattgefundenen Streit nicht kennen. Eine Ausnahme bilden die beiden zuvor herausgestellten Positionen von Kirchgässner und Vanberg, deren Beiträge zum Methodenstreit eine interessante Schnittmenge aufweisen: Beide erachten Mathematik als Instrument, dessen Einsatz für den Erkenntnisgewinn lohnend ist. Beiden Beiträgen ist ebenfalls eine kategorische Ablehnung von Wissens- und Werturteilsanmaßung zu Eigen, indem sich beide, wenngleich auch unterschiedlich argumentierend, auf das Wertfreiheitspostulat im Sinne von Hans Albert (1963) beziehen.

Die Frage nach der methodischen Ausrichtung der Ökonomik, insbesondere der Ordnungsökonomik, hat viele Facetten. Natürlich wird ein Argument nicht dadurch überzeugend, dass es formal mathematisch vorgebracht wird. Aber die Mathematik ist eine Sprache, die aufgrund ihrer logischen Stringenz sehr viele Vorteile dahingehend hat. Sie zwingt Wissenschaftler, ihre Argumente auf Widerspruchsfreiheit und Konsistenz zu überprüfen sowie die Voraussetzungen für die Gültigkeit ihrer Hypothesen genau zu benennen. Es gilt jedoch, dass ein gutes Argument nicht notwendigerweise mathematisch vorgebracht werden muss. Es lässt sich trefflich ohne Mathematik theoretisieren. Die Methode der Analytical Narratives ist wohl etabliert. Allerdings schreckt die Mathematik manche Rezipienten ab. Ökonomische Argumentation wird für andere Menschen unzugänglich und die Ökonomik droht, ihre gesellschaftliche Relevanz, insbesondere im Zusammenspiel mit ihren Nachbardisziplinen, zu verlieren. Will die Ökonomik ihre Bringschuld gegenüber der Gesellschaft einlösen, sollten Ökonomen in der Lage sein, ihre Argumente auch allgemein verständlich vorzubringen. Dennoch muss nicht jeder Ökonom über solche Fähigkeiten verfügen; Arbeitsteilung trägt hier ebenso zu gesellschaftlichen Verbesserungen bei wie in anderen ökonomischen $\mathrm{Zu}$ sammenhängen. Für jede Form der Theorie in den Realwissenschaften, die sich mit lebensweltlichen Phänomenen im weiteren Sinne befassen, ist es jedoch notwendig, im Popper'schen Sinne falsifizierbar zu bleiben und gleichzeitig am Wissenschaftsmarkt bestreitbar zu sein. Gerade für eine überzeugende empirische Prüfung von Hypothesen sind mathematisch-statistische Methoden sehr hilfreich. Obwohl die Komplexität wirtschaftlicher Phänomene hinsichtlich der empirischen Analyse zu Vorsicht und Bescheidenheit mahnt, bleibt die empirische Überprüfung ordnungsökonomischer Hypothesen eine zentrale Herausforderung für die Ordnungsökonomik.

Das Wertfreiheitspostulat verstärkt diese Argumente. Es macht wenig Sinn, die Wertfreiheit beim einzelnen Wissenschaftler, etwa noch mit moralischem Anspruch einzufordern. Wissenschaftler haben eigene Wertvorstellungen und es ist nicht zu erwarten, dass sie diese in ihrer Forschung unberücksichtigt lassen. Dies beginnt bereits mit der Auswahl der Forschungsthemen und -projekte, geht über die Wahl der Methoden bis hin zu den Forschungsergebnissen. Selbst bei der Erhebung von Daten fließen Wertvorstellungen ein. Die Wertfreiheit der Wissenschaft resultiert somit nicht aus der moralisch eingeforderten Wertfreiheit des einzelnen Wissenschaftlers. Vielmehr ist sie Resultat des Wissenschaftsprozesses. Ist die Wissenschaft ein offenes System, so sind wissenschaftliche Untersuchungen bestreitbar. Wissenschaftler, welche die mit ihren Werthaltungen erzielten Forschungsergebnisse präsentieren, müssen im wissenschaftlichen Wettbewerb bestehen. Ihre Ergebnisse unterliegen der potentiellen Kritik anderer Wissenschaftler und sie laufen Gefahr, ihre Reputation zu verlieren, wenn sie ihre 
Wertvorstellungen in den Vordergrund stellen. Da diese Reputation einen hohen Wert hat, stellen sich Wissenschaftler schon im Vorfeld auf mögliche Kritikpunkte ein. Der potentielle Reputationsverlust beschränkt das Ausmaß, in welchem sie ihr Werturteil in ihrer Forschung zum Tragen bringen können. Der Wettbewerb unter Wissenschaftlern führt dann dazu, dass sich wissenschaftliche Ergebnisse vorläufig bewähren, soweit sie nicht falsifiziert werden. Dadurch werden wissenschaftliche Ergebnisse von den Werturteilen der sie hervorbringenden Wissenschaftler unabhängig.

Die mit einem solchen offenen Wissenschaftssystem verbundene Hoffnung auf vernünftige und verlässliche Ergebnisse soll nicht den Blick dafür verstellen, dass es Fehlentwicklungen in der Wissenschaft gibt. Der Mainstream beherrscht in der Regel die Akzeptanz von Forschungsmethoden und -ergebnissen. Gewagte, vom Mainstream stark abweichende Ansätze haben es schwer. Heterodoxe Wissenschaft ist selten beliebt. Sie steht am Rande. Dadurch halten sich traditionelle Forschungsansätze länger als es zuweilen akzeptabel erscheint. Allerdings spielen Moden durchaus eine Rolle. So führte die experimentelle Ökonomik bereits in den 1960er Jahren zu sehr beachtlichen Ergebnissen, begann aber erst im vergangenen Jahrzehnt die mikroökonomische Forschung in referierten internationalen Fachzeitschriften zu dominieren. Zudem kann die Organisation des Wissenschaftsprozesses selbst bei großer Offenheit des Systems zu Fehlsteuerungen führen. Die starke Orientierung an Zeitschriftenrankings in der Ökonomik belegt dies. Einerseits führt ein solcher international anerkannter Maßstab wissenschaftlicher Leistungen dazu, dass das Wissenschaftssystem insbesondere international offen ist und frühere Mechanismen zur Bewertung der Fähigkeit, selbständig zu lehren und zu forschen, in den deutschen Wirtschaftswissenschaften neben dem formalen Kriterium der Habilitation nicht selten die Zugehörigkeit zu bestimmten Schulen, zurückgedrängt werden. Andererseits führen diese Rankings zu Übertreibungen, wie etwa der Tendenz, eine Idee durch Orientierung an der least publishable unit extrem zu portionieren und damit möglichst viele Veröffentlichungen in Zeitschriften zu erhalten, oder indem man alles tut, um im Begutachtungsprozess zu reüssieren (Frey 2003).

Der für die Weiterentwicklung der Ordnungsökonomik bedeutsamste Schritt stellt aber weder die methodische Orientierung noch die damit verbundene Forderung dar, sich dem Wettbewerb der Ideen aktiv zu stellen und sich damit dem Zwang zu unterwerfen, Werturteile offen zu legen. Wichtiger erscheint die sich anhand der Gegenüberstellung von Kirchgässners (1988) Kritik an der Ordnungsökonomik und Vanbergs Bemühungen zu ihrer Weiterentwicklung herausstellende Kongruenz der Anliegen der Ordnungs- und Verfassungsökonomik. Die Nähe zur Verfassungsökonomik und zur Neuen Politischen Ökonomik unterstreicht die Zukunftsfähigkeit der modernen Ordnungsökonomik.

\section{Beiträge zur Weiterentwicklung der Ordnungsökonomik}

Nach dem Methodenstreit von 2009 hat sich die Diskussion um die Zukunft der Ordnungsökonomik fortgesetzt. Dabei sind zwei Richtungen zu beobachten, nämlich einerseits die Erweiterung des normativen Ansatzes in wirtschafts- und sozialethischer Absicht und andererseits die Vorschläge, sich nach einer strikten Trennung der Ord- 
nungsökonomik in eine normative und positive Forschungsrichtung auf den positiven Zweig zu konzentrieren.

Goldschmidt (2011) versucht die Ordnungsökonomik zu modernisieren, indem er die normativen Grundlagen der Gesellschaft offen legt, die in der wirtschaftsethischen Chiffre der Freiburger Schule seines Erachtens enthalten sind (Goldschmidt 2011: 145ff.). Ausgehend von der in der Glücksforschung vorherrschenden Fragestellung, welche Faktoren das Glück der Menschen beeinflussen und wie dieses zu erhöhen ist, weist Goldschmidt (2011) auf die Gefahr hin, dass die Glücksforschung einem „naturalistischen Fehlschluss“ unterliegt. Wenn aus dem „Ist-Zustand des empirischen Wissens über einzelne Faktoren, die dem Wohlbefinden Einzelner zuträglich sind“, geschlossen wird, „dass die Faktoren auch befördert werden sollten“, liege eine diffuse utilitaristische Legitimation vor, an deren Stelle eine adäquate normative Fundierung rücken sollte (Goldschmidt 2011: 149). Für diese normative Fundierung müsse man zunächst feststellen, „welche normative Leitidee für moderne Gesellschaften tragfähig ist", auch um die „moderne Ordnungsökonomik selbst klarer zu fassen“ (Goldschmidt 2011: 150). Damit stellt Goldschmidt der Glücksforschung [s] eine ordnungsökonomische Perspektive voran, allerdings mit Hilfe eines „vitalpolitischen“ Paradigmas und nicht, indem er im Buchanan'schen Sinne eine direkte Rückbindung auf die konsensfähigen konstitutionellen Interessen der Bürger oder die Steuerungsideale der Konsumenten- und Bürgersouveränität vornimmt. Basierend auf externen Werturteilen - die Sen'sche Ordnungsethik - erkennt er in der Vitalpolitik eine ,gesellschaftspolitische Aufgabe“, die eine „bewusste[n] politische und qualitative Förderung von Lebensumständen“ zum Ziel hat, um „dem Einzelnen Lebensperspektiven“ zu eröffnen (Goldschmidt 2011: 151).

Im Weiteren fordert er den „Aufbau einer [...] gesellschaftlichen Ordnung, die jedem Mitglied der Gesellschaft die Möglichkeit gibt, „frei und einen nach den jeweiligen Fähigkeiten und Zielsetzungen bestimmten Lebensweg zu wählen. Es geht also nicht so sehr um die Herstellung von (Glücks-) Zuständen, sondern um die Ermöglichung von Bedingungen eines sinnerfüllten Lebens“ (Goldschmidt 2011: 152). Aus verfassungsökonomischer Perspektive könnte es durchaus gute Gründe geben, eine solche Regel - oder ein Leitideal für die Sozialpolitik - zu empfehlen. Allerdings fehlt dieser Analyse eine ausführliche Erörterung der Klugheitsargumente, die sich für und gegen ein solches Arrangement ins Feld führen lassen. Problematisch sind zudem die an diese Stelle rückenden historisierenden Argumentationen, die an den von Kirchgässner diskutierten Ideologievorwurf erinnern: „Dergestalt handelt es sich um ein klar ordoliberales Konzept im Sinne der Freiburger Tradition" (Goldschmidt 2011: 15). Argumente für eine bestimmte Sozialordnung müssen ohne den Rekurs auf die Tradition überzeugen. An diesem Punkt setzt aber sein Beitrag mit Alexander Lenger an (2011: 3): ,[...] die Mitglieder eines Gemeinwesen [entscheiden] frei über das jeweilige soziale Arrangement, und zwar dergestalt, dass sie sich auf eine gesellschaftliche Ordnung einigen, die im konstitutionellen Interesse aller beteiligten Individuen liegt.“ Hier wird aus dem Legitimationsprinzip der freiwilligen Zustimmung die Forderung nach einer diskriminierungsfreien „Sozial“-Ordnung als prozedurales Kriterium abgeleitet. 
Zwar erscheint diese Argumentation schließlich normativ individualistisch. Gleichwohl bleiben die Autoren hinter Vanbergs Ansatz zur Modernisierung der Ordnungsökonomik zurück, weil nicht eindeutig auf das Konzept der Bürgersouveränität abgestellt und stattdessen ein sozialpolitisches Paradigma der Ordnungsökonomik vorangestellt wird: Hier greifen die Argumente zu kurz, wenn nicht deutlich wird, mit Hilfe welcher Institutionen die diskriminierungsfreie Ordnung errichtet werden soll. Das Konzept von Goldschmidt und Lenger (2011) benötigt irgendeine Instanz, die darüber wacht, dass tatsächlich alle Bürger in einer diskriminierungsfreien Ordnung leben. Damit sind Ausgestaltungsprobleme direkt vorprogrammiert.

Schließlich zielen die unterschiedlichen von Goldschmidt und Koautoren verfassten Beiträge darauf ab, Inklusion und „Teilhabe“ zu einem Steuerungsideal und prozeduralen Kriterium zu erheben, welche jedoch in Konflikt zur Bürger- und Konsumentensouveränität geraten können, wenn sie uneingeschränkt ausgestaltet werden (Goldschmidt 2011a). Damit bewegen sich die Beiträge zwischen Rawls (1975) und der christlichen Sozialethik und lassen sich nur unzureichend konkret auf die Bürgersouveränität ein, unterschätzen die zu erwartenden politökonomischen Konsequenzen oder machen Vorgaben für die Ordnungsethik auf Basis exogener Normen. Vermutlich sollte Goldschmidts Inklusionsidee einem Zustimmungstest unterworfen werden, jedoch nicht an die Stelle eines solchen rücken.

Einen Ausweg könnte der positive verfassungsökonomische Ansatz von Voigt (1997, 2011) bieten. Er unterscheidet zwei Kernforschungsgebiete, die sich der ökonomischen Analyse von Verfassungen annehmen: Erstens einer normativen Richtung, die entlang des Forschungsprogramms von Buchanan (1987) fragt, wie Verfassungsregeln prinzipiell zu legitimieren sind und dabei dem normativen und methodologischen Individualismus verhaftet bleibt; zweitens einer positiven Richtung, welche die ökonomischen Auswirkungen alternativer Regeln bzw. die Entstehung und Variation von Verfassungsregeln untersucht. Unterschiede in den wirtschaftspolitischen Ergebnissen lassen sich zwischen verschiedenen politischen Systemen - Demokratie vs. Diktatur, direkte vs. repräsentative Demokratie, Mehrheits- vs. Verhältniswahlrecht, Präsidialvs. parlamentarische Demokratie, Einheits- vs. Bundesstaat - empirisch feststellen (Voigt 2011). Die Bestimmungsgründe für die Entstehung bestimmter Verfassungsregeln sind jedoch empirisch wenig erforscht. Hier verortet Voigt (2011) den größten Handlungsbedarf, um künftig - insbesondere mit Hilfe der experimentellen Ökonomik - neue Erkenntnisse über das Verhalten rationaler Akteure zu gewinnen.

Voigt $(1997,2011)$ bleibt relativ vage hinsichtlich der Implikationen seiner Analyse für die moderne Ordnungsökonomik. Es scheint gleichwohl an verschiedenen Stellen dieser beiden Übersichtsaufsätze auf, dass er eindeutige Vorzüge der positiven gegenüber der normativen Verfassungsökonomik sieht. Für die Ordnungspolitik gehe es darum, die Lücke zwischen Sein und Sollen weitgehend zu schließen, indem das Sollen, der Rekurs auf normative Vorgaben, immer weiter zurückgedrängt wird. Dass dieser Gegensatz nicht in dieser Schärfe besteht, wenn man wie Vanberg auf das aus dem normativen Individualismus gewonnene Konzept der Bürgersouveränität zurückgreift, berücksichtigt Voigt nicht. Gleichwohl bietet seine Perspektive auf die Verfassungsökonomik die Konkretisierung an, die bei Goldschmidt fehlt. 


\section{Schlussbemerkungen: Zur Zukunftsfähigkeit der Ordnungsökonomik}

Die Zukunftsfähigkeit der Ordnungsökonomik als eigenständiges Forschungsprogramm der Wirtschaftswissenschaften erschließt sich durch ihre Kongruenz mit der Verfassungsökonomik. Zwar ist die traditionelle Ordnungsökonomik in ihren verschiedenen Verästelungen und Dimensionen vielfältig (Eucken, Müller-Armack, Röpke, Rüstow). Auch lehnten Eucken und Böhm die Ausdehnung der ökonomischen Analyse auf den Datenkranz von rechtlichen und politischen Rahmenbedingungen der Wirtschaft mehr oder weniger stark ab. Die Freiburger Schule der Ordnungsökonomik dürfte gleichwohl aufgrund ihrer Regelorientierung keine Schwierigkeiten mit dem Prinzip der Zustimmungsfähigkeit als Legitimationskriterium und den Steuerungsidealen der Konsumenten- und Bürgersouveränität gehabt haben. Basierend auf dem normativen und methodologischen Individualismus deckt sich eine so verstandene Ordnungsökonomik mit der Buchanan'schen Verfassungsökonomik.

Die Ordnungsökonomik kann theoretisch und empirisch unter Berücksichtigung des institutionellen Umfeldes untersuchen, wie gemeinsame Bürgerinteressen berücksichtigt werden können, um eine reagible demokratische Wirtschafts- und Staatsordnung auszugestalten. Diese Neuorientierung kann vom Ausgangspunkt der Rawls'schen Primärdefinition von Demokratie begonnen werden, wenn man ein demokratisches Gemeinwesen ,als ein Unternehmen der Zusammenarbeit zum gegenseitigen Vorteil“ versteht (Rawls 1975: 105). In diesem Sinne zeichnet sich das demokratische Gemeinwesen als ein „genossenschaftlicher, mitgliederbestimmter Verband“ aus, der dem ,wechselseitigen Vorteil seiner Mitglieder“, den Bürgern, dienen soll - oder kurz „der Bürgergenossenschaft“ (Vanberg 2004b: 118): Der wesentliche Unterschied zwischen Primär- und Sekundärdefinitionen besteht darin, dass die Frage, welche institutionellen Regelungen am besten geeignet sind, zum wechselseitigen Vorteil der Mitglieder der Bürgergenossenschaft zu wirken, aus „Sicht der Primärdefinition eine empirisch und theoretisch zu klärende Faktenfrage darstellt“", während „Sekundärdefinitionen dazu tendieren, durch ihre definitorischen Festlegungen diese Frage empirischer Prüfung und rationaler Diskussion zu entziehen." Sekundärdefinitionen von Demokratie stellen auf bestimmte institutionelle oder organisatorische Charakteristika $\mathrm{ab}$, während Primärdefinitionen noch nichts über die konkrete institutionelle Ausgestaltung des genossenschaftlichen Verbandes aussagen.

Auf Letzteres sollte die neue Ordnungsökonomik großen „Wert“ legen, wenn es das Ziel darstellt, sie weder ideologisch noch entlang des Status Quo - oder rein im Rekurs auf die alten Denker der Freiburger Schule - zu begründen. Folgt man der Primärdefinition und versteht die Demokratie in erster Linie als Bürgergenossenschaft, die wechselseitige Vorteile ihrer Bürger zu suchen hat, setzt man den weiteren Regelrahmen zur gestalterischen Disposition. Geht man diesen Weg von der Primärdefinition der Demokratie über die Verfassungsökonomik zum Methodenstreit bis zum Ende, bietet sich die Bürgersouveränität nicht nur als Verfahrenskriterium an, entlang dessen die Tragfähigkeit der wirtschaftspolitischen Ausgestaltung der Ordnungsökonomik erörtert werden kann, sondern auch als Verpflichtung, bestehende institutionelle Arrangements im Sinne der Bürgersouveränität zu reformieren - und darüber hinaus neue Institutionen zu suchen, die die Stellung des Bürgers als Souverän stärken. 
Damit ist nicht nur gemeint, sich für mehr föderalen Wettbewerb stark zu machen (ob auf europäischer oder bundesstaatlicher Ebene), Freizügigkeits- bzw. Migrationsregelungen unter diesem Leistungskriterium neu auszugestalten, sondern auch jenseits der Ordnungsrealität nach alternativen Arrangements zu suchen, die gemeinsame Kooperationsvorteile für den Bürger ermöglichen.

Wird die Ordnungsökonomik entlang des Kriteriums der Bürgersouveränität neu ausgerichtet, wird die alte freiheitliche Grundidee erneut in den Vordergrund gerückt, den Bürger zum Subjekt des politischen Prozesses und zum Souverän des Gemeinwesens zu ermächtigen: Schließlich werden diejenigen institutionellen Arrangements unter der Anwendung des Steuerungsideals der Bürgersouveränität empfohlen, die eine höhere Reagibilität auf Bürgerinteressen erwarten lassen. Solche institutionellen Arrangements müssen im Sinne eines positiven Forschungsprogramms identifiziert werden, bevor sie durch Rückbindung an den normativen Individualismus zur Verfolgung des Bürgerinteresses nahe gelegt werden können. Theoretische Erkenntnisse müssen somit empirisch fundiert sein. Für diese Identifikation sind mathematischstatistische Methoden notwendig. Trotz der Komplexität wirtschaftlicher Phänomene lässt ein solches Vorgehen, wenn es mit aller Bescheidenheit durchgeführt wird, auf den wissenschaftlichen Fortschritt der ordnungsökonomischen Forschung hoffen.

\section{Literaturverzeichnis}

Albert, H. (1963): Wertfreiheit als methodisches Prinzip: Zur Frage der Notwendigkeit einer normativen Sozialwissenschaft, in: von Beckerath, E./ Giersch, H. (Hrsg.): Probleme der normativen Ökonomik und der wirtschaftspolitischen Beratung, Berlin: Duncker \& Humblot, 32-63, wiederabgedruckt in: Topitsch, E. (Hrsg.): Logik der Sozialwissenschaften, Köln: Kiepenheuer \& Witsch 1965, 181-210.

Bachmann, R. (2011): Zum Neueren Methodenstreit - Rückblick und Ausblick, in: Caspari, V./ Schefold, B. (Hrsg.): Wohin steuert die ökonomische Wissenschaft? - Ein Methodenstreit in der Volkswirtschaftslehre, Frankfurt a. M.: Campus, 259-268.

Bachmann, R./ Ublig, H. (2009): Die Welt ist nicht schwarz und weiß, Frankfurter Allgemeine Zeitung vom 29.03.2009.

Böhm, F. (1957): Die Forschungs- und Lehrgemeinschaft zwischen Juristen und Volkswirten an der Universität Freiburg in den dreißiger und vierziger Jahren des 20. Jahrhunderts, in: Wolff, H. J. (Hrsg.): Aus der Geschichte der Rechts- und Staatswissenschaften zu Freiburg i. Br., Freiburg: E. Albert, 95-113.

Böhm, F. (1973): Eine Kampfansage an Ordnungstheorie und Ordnungspolitik, ORDO - Jahrbuch für die Ordnung von Wirtschaft und Gesellschaft 24, 11-48.

Bränninger, M./ Haucap, J./ Muck, J. (2011): Was lesen und schätzen Ökonomen im Jahr 2011? Ordnungspolitische Perspektiven Nr. 18, Düsseldorfer Institut für Wettbewerbsökonomie (DICE), Heinrich Heine Universität Düsseldorf.

Brennan, G./ Buchanan, J. M. (1985): The Reason of Rules, Cambridge University Press, Cambridge; zitiert nach: The Collected Works of James M. Buchanan, Vol. 10, Indianapolis: Liberty Fund, 2000.

Buchanan, J. M. (1975): The Limits of Liberty: Between Anarchy and Leviathan, Chicago: University of Chicago Press. 
Buchanan, J. M. (1984): Constitutional Restrictions on the Power of Government, in: Buchanan, J. M./ Tollison, R. D. (Hrsg.): The Theory of Public Choice II, Ann Arbor: The University of Michigan Press.

Buchanan, J. M. (1987): The Constitution of Economic Policy, American Economic Review 77, 243-250.

Buchanan, J. M. (2010): Chicago School Thinking: Old and New, Unveröffentlichtes Manuskript, Center for the Study of Public Choice, Fairfax, http://jepson.richmond.edu /conferences/adam-smith/paper2010buchanan.pdf.

Caspari, V./ Schefold, B. (2011): Wohin steuert die ökonomische Wissenschaft? - Ein Methodenstreit in der Volkswirtschaftslehre, Frankfurt a. M.: Campus.

Cassel, D./ Ramb, B.-T./ Thieme, H. J. (1988): Vorwort, in: Cassel, D./ Ramb, B.-T./ Thieme, H. J. (Hrsg.): Ordnungspolitik, München: Vahlen, 7.

Dathe, U. (2010): Walter Euckens Weg zum Liberalismus, Freiburger Diskussionspapiere zur Ordnungsökonomik 09/10.

Eucken, W. A. (1932): Staatliche Strukturwandlungen und die Krisis des Kapitalismus, Weltwirtschaftliches Archiv 36, 297-321.

Eucken, W. A. (1940): Grundlagen der Nationalökonomie, 7. Auflage 1959, Berlin et al.: Springer.

Eucken, W. (1952): Grundsätze der Wirtschaftspolitik, Tübingen: Mohr.

Feld, L. P./ Kirchgässner, G. (2009): Wirkungen direkter Demokratie - Was sagt die moderne politische Ökonomie? In: Heußner, H. K./ Jung, O. (Hrsg.): Mehr direkte Demokratie wagen - Volksentscheid und Bürgerentscheid: Geschichte - Praxis - Vorschläge, 2. Auflage, München: Olzog, 417-430.

Frey, B. S. (1981): Theorie demokratischer Wirtschaftspolitik, München: Vahlen.

Frey, B. S. (2003): Publishing as Prostitution, Public Choice 116, 205-223.

Fukuyama, F. (1989): The End of History? In: Fukuyama, F.: The National Interest, New York: Free Press.

Giersch, H. (1993): Was es heißt, Volkswirt zu sein, in: Giersch, H.: Marktwirtschaftliche Perspektiven für Europa, Düsseldorf: Econ, 20-44.

Giersch, H. (1994): Thesen zum Thema Wissenschaftler in der wirtschaftspolitischen Verantwortung, in: Orden pour le mérite für Wissenschaften und Künste (Hrsg.): Reden und Gedenkworte, Vierundzwanzigster Band 1993 - 1994, Gerlingen: Verlag Lambert Schneider, 241-246.

Goldschmidt, N. (2011): Vom Glück und vom Gärtner - Moderne Ordnungsökonomik und die normativen Grundlagen der Gesellschaft, in: Caspari, V./ Schefold, B. (Hrsg.): Wohin steuert die ökonomische Wissenschaft? - Ein Methodenstreit in der Volkswirtschaftslehre, Frankfurt a. M.: Campus, 145-166.

Goldschmidt, N. (2011a): Kapitalismus mit menschlichem Antlitz. Wirtschaftsliberalismus und katholische Soziallehre: aktuelle und historische Spuren, Schweizer Monat, Sonderthema 1, September, 6-8.

Goldschmidt, N./ Lenger, A. (2011): Teilhabe und Befähigung als Schlüsselelemente einer modernen Ordnungsethik, in diesem Heft.

Goldschmidt, N./ Wegner, G./ Woblgemuth, M./ Zweynert, J. (2009): Was ist und was kann Ordnungsökonomik? Frankfurter Allgemeine Zeitung vom 19.06.2009.

Haucap, J. (2009): Nachtrag: Krise der Wirtschaftswissenschaften: Braucht die VWL eine Neuausrichtung? ifo Schnelldienst Vol. 62/ No. 15, 19-22. 
Hayek, F. A. von (1960): The Constitution of Liberty, Chicago: University of Chicago Press.

Hayek, F. A. von (1967): Rechtsordnung und Handelnsordnung, in Streißler, E. (Hrsg.): Zur Einheit der Rechts- und Staatswissenschaften, Karlsruhe: C. F. Müller, 195-230; wiederabgedruckt in: Hayek, F. A. (2003): Rechtsordnung und Handelnsordnung: Aufsätze zur Ordnungsökonomik, Gesammelte Schriften in deutscher Sprache, Abt. A, Band 4, Tübingen: Mohr Siebeck, 35-73.

Hicks, J. R. (1974): The Crisis in Keynesian Economics, Oxford: Blackwell.

Hoppmann, E. (1987): Ökonomische Theorie der Verfassung, ORDO - Jahrbuch für die Ordnung von Wirtschaft und Gesellschaft 38, 31-45.

Kirchgässner, G. (1988): Wirtschaftspolitik und Politiksystem: Zur Kritik der traditionellen Ordnungstheorie aus der Sicht der Neuen Politischen Ökonomie, in: Cassel, D./ Ramb, B.T./ Thieme, H. J. (Hrsg.): Ordnungspolitik, München: Vahlen, 53-75.

Kirchgässner, G. (2009): Die Krise der Wirtschaft: Auch eine Krise der Wirtschaftswissenschaften? Perspektiven der Wirtschaftspolitik 10, 436-468.

Kirchgässner, G. (2009a): Der Rückzug ins nationale Schneckenhaus, Frankfurter Allgemeine Sonntagszeitung vom 15.06.2009.

Kirchgässner, G./ Feld, L. P./ Savioz, M. R. (1999): Die direkte Demokratie: Modern, erfolgreich, entwicklungs- und exportfähig, Basel/München: Helbing und Lichtenhahn/Vahlen.

Krugman, P. (2009): How did Economists Get it So Wrong? New York Times vom 2.09.2009, S. 36, http://www.nytimes.com/2009/09/06/magazine/06Economic-t.html.

Krugman, P. (2011): The President Surrenders, New York Times vom 1.08.2011, S. A21, http://www.nytimes.com/2011/08/01/opinion/the-president-surrenders-on-debtceiling.html (Download am 9.08.2011).

Leipold, H. (1988): Ordnungspolitische Konsequenzen der ökonomischen Theorie der Verfassung, in: Cassel, D./ Ramb, B.-T./ Thieme, H. J. (Hrsg.): Ordnungspolitik, München: Vahlen, 257-284.

Lenel, H.-O. (1971): Haben wir noch eine soziale Marktwirtschaft? ORDO - Jahrbuch für die Ordnung von Wirtschaft und Gesellschaft 22, 29-47.

Molitor, B. (1982): Schwäche der Demokratie, ORDO - Jahrbuch für die Ordnung von Wirtschaft und Gesellschaft 34, 17-38.

Pierce, A. (2008): The Queen Asks Why No One Saw the Credit Crunch Coming, Daily Telegraph vom 5.11.2008, http://www.telegraph.co.uk/news/uknews/theroyalfamily/ 3386353/The-Queen-asks-why-no-one-saw-the-credit-crunch-coming.html.

Plickert, P. (2009): Der Streit unter den Ökonomen eskaliert, Frankfurter Allgemeine Zeitung vom 4.06.2009.

Popper, K. R. (1957): Die offene Gesellschaft und ihre Feinde, Bd. 1, Der Zauber Platons, Francke, Bern; 7. Auflage, Tübingen: Mohr Siebeck 1992.

Rawls, J. (1975): Eine Theorie der Gerechtigkeit, Frankfurt a. M.: Suhrkamp.

Richter, R. (1987): Geldtheorie: Vorlesung auf der Grundlage der Allgemeinen Gleichgewichtstheorie und der Institutionenökonomik, 2. Aufl. 1990, Springer: Berlin.

Richter, R. (1988): The New Institutional Economics Applied to Monetary Economics, Journal of Institutional and Theoretical Economics 144, 208-224.

Rowley, C. K. (1982): The Failure of Government to Perform its Proper Task, ORDO - Jahrbuch für die Ordnung von Wirtschaft und Gesellschaft 34, 39-58.

Schirmacher, F. (2010): Vorwort, in: Schirrmacher, F./ Strobl, Th. (Hrsg.): Die Zukunft des Kapitalismus, Berlin: Suhrkamp. 
Schmidt, C./ aus dem Moore, N. (2009): Quo vadis, Ökonomik? Frankfurter Allgemeine Zeitung vom 22.05.2009.

Sinn, H. W. (2009): Der richtige Dreiklang der VWL, Frankfurter Allgemeine Zeitung, Nr. 141, 22.06.2009, 12 .

Streit, M. (1996): Ordnungsökonomik, in: Gabler-Volkswirtschafts-Lexikon, Wiesbaden: Gabler, 814-843.

Streit, M./ Woblgemuth, M. (2000): Walter Eucken und Friedrich A. von Hayek: Initiatoren der Ordungsökonomik, in: Külp, B./ Vanberg, V. J. (Hrsg.): Freiheit und wettbewerbliche Ordnung - Gedenkband zur Erinnerung an Walter Eucken, Freiburg: Haufe.

Turley, H. (1961): Neoliberale Monopoltheorie und „Antimonopolismus“ - Ein Beitrag zur Aufdeckung des apologetischen Charakters des Neoliberalismus in Westdeutschland, Berlin: Akademie-Verlag.

Vanberg, V.J. (1988): 'Ordnungstheorie' as Constitutional Economics. The German Conception of a 'Social Market Economy', ORDO - Jahrbuch für die Ordnung von Wirtschaft und Gesellschaft 39, 17-31.

Vanberg, V. J. (1994): Rules and Choice in Economics, London: Routledge.

Vanberg, V.J. (1997): Die normativen Grundlagen von Ordnungspolitik, ORDO - Jahrbuch für die Ordnung von Wirtschaft und Gesellschaft 48, 707-726; wiederabgedruckt in: Vanberg, V. J. (2008): Wettbewerb und Regelordnung, Tübingen: Mohr Siebeck, 49-67.

Vanberg, V. J. (1998): Constitutional Political Economy, in: Davis, J. B./ Hands, D. W./ Mäki, U. (Hrsg.): The Handbook of Economic Methodology, Cheltenham: Edward Elgar, 69-75.

Vanberg, V. J. (2004): The Freiburg School: Walter Eucken and Ordoliberalism, Freiburger Diskussionspapiere zur Ordnungsökonomik 11/2004.

Vanberg, V. J. (2004a): Market and State: The Perspective of Constitutional Political Economy, Freiburger Diskussionspapiere zur Ordnungsökonomik 10/2004.

Vanberg, V. J. (2004b): Bürgersouveränität und wettbewerblicher Förderalismus: Das Beispiel der EU, in: Schäfer, W. (Hrsg.): Zukunftsprobleme der europäischen Wirtschaftsverfassung, Berlin: Duncker \& Humblot, 51-86; wiederabgedruckt in: Vanberg, V. J. (2008): Wettbewerb und Regelordnung, Tübingen: Mohr Siebeck, 117-151.

Vanberg, V. J. (2009): Die Ökonomik ist keine zweite Physik, Frankfurter Allgemeine Zeitung vom 13.04.2009.

Vanberg, V. J. (2011): Liberal Constitutionalism, Constitutional Liberalism and Democracy, Constitutional Political Economy 22, 1-20.

Vaubel, R. (2011): Rettet die Volkswirtschaftslehre an den Universitäten: Zum Aufruf der 83 VWL-Professoren, in: Caspari, V./ Schefold, B. (Hrsg.): Wohin steuert die ökonomische Wissenschaft? - Ein Methodenstreit in der Volkswirtschaftslehre, Frankfurt a. M.: Campus, 269-273.

Voigt, S. (1997): Positive Constitutional Economics - A Survey, Public Choice 90, 11-53.

Voigt, S. (2011): Positive Constitutional Economics II - A Survey of Recent Developments, Public Choice 146, 205-256.

Willgerodt, H. (2009): Von der Wertfreiheit zur Wertlosigkeit, Frankfurter Allgemeine Zeitung, Nr. 49, 27.02.2009, 12 .

Wissenschaftlicher Beirat beim Bundesministerium für Wirtschaft und Technologie (2009): Akzeptanz der Marktwirtschaft: Einkommensverteilung, Chancengleichheit und die Rolle des Staates, Gutachten, Berlin. 